\title{
Leaf Rust Resistance Gene Lr34 Associated with Nonsuppression of Stem Rust Resistance in the Wheat Cultivar Canthatch
}

\author{
E. R. Kerber and T. Aung
}

Agriculture and Agri-Food Canada, Cereal Research Centre, 195 Dafoe Road, Winnipeg, MB, Canada R3T 2M9.

Accepted for publication 22 February 1999.

\begin{abstract}
Kerber, E. R., and Aung, T. 1999. Leaf rust resistance gene Lr34 associated with nonsuppression of stem rust resistance in the wheat cultivar Canthatch. Phytopathology 89:518-521.

The common wheat cultivar Thatcher and the backcross derivative Canthatch are moderately or fully susceptible to several races of stem rust because of a suppressor on chromosome 7DL that inhibits the expression of the relevant resistance gene(s). The incorporation of leaf rust resistance gene Lr34 into 'Thatcher' is known to enhance stem rust resistance.

short arm. 'Canthatch' nullisomic 7D was crossed with 'Chinese Spring', followed by a succession of backcrosses to the nullisomic recurrent parent. Homozygous resistant disomic and monosomic substitution lines were recovered that exhibited the same resistant reaction as that of 'Thatcher' possessing $L r 34$ and as that of 'Canthatch' nullisomic 7D, in which the 7DL suppressor is absent. The results indicate that, in 'Canthatch', Lr34 permits expression of resistance genes normally inhibited by the 7DL suppressor. Furthermore, it is likely that, in 'Thatcher' and 'Thatcher' backcross derivatives, $\operatorname{Lr} 34$ inactivates the 7DL suppressor.
\end{abstract} The effect of this gene in a 'Canthatch' background and its relationship with the 7DL suppressor were determined by replacing chromosome 7D of 'Canthatch' with 7D of 'Chinese Spring', which possesses Lr34 on the
Additional keywords: Puccinia graminis f. sp. tritici, Puccinia recondita f. sp. tritici, Triticum aestivum, wheat leaf rust.
The common wheat cultivar Canthatch (Triticum aestivum L.) possesses a suppressor gene on the long arm of chromosome 7D that inhibits the expression of resistance to several races of stem rust (Puccinia graminis Pers. f. sp. tritici Eriks. \& E. Henn.). The resistance is expressed in the absence of the long arm (7DL) as in 'Canthatch', which is ditelosomic for 7DS or nullisomic for 7D $(9,11)$. A nonsuppressing mutation induced on chromosome arm 7DL of 'Canthatch' also results in the expression of resistance to these races $(9,10)$. Although no wheat cultivar has been identified that possesses a nonsuppressing allele of the 7DL suppressor, backcross derivatives of 'Thatcher', into which the leaf rust ( $P$. recondita Roberge ex Desmaz. f. sp. tritici) resistance gene Lr34 had been transferred, exhibited enhanced resistance to races of stem rust to which both 'Thatcher' and 'Canthatch' were susceptible $(1,3,6,13)$. For example, 'Thatcher' with Lr34 exhibited seedling infection type (IT) 0 ; to each of four races of stem rust, whereas 'Thatcher' gave IT $2^{+} 3^{+}$to one of these and IT $3^{+}$to each of the other three (13). Since 'Canthatch' is a backcross derivative of 'Thatcher', the latter likely is the donor of the 7DL suppressor and also of the inhibited resistance gene(s). It is conceivable, therefore, that, in a 'Thatcher' background, Lr34 or associated chromatin nullifies the effect of the 7DL suppressor and results in greater stem rust resistance because of the expression of one or more previously inhibited, unidentified resistance genes on chromosomes other than 7D. Lr34 itself, located on the short arm of chromosome 7D $(5,15)$, confers no resistance to stem rust (1). 'Thatcher' and 'Canthatch' possess stem rust resistance genes $\mathrm{Sr} 5, \mathrm{Sr} 9 \mathrm{~g}, \mathrm{Sr} 12$, and $\mathrm{Srl6}$ (14). In addition, 'Canthatch' possesses $\operatorname{Sr} 7 a$ (11). These genes are ineffective against the races to which 'Thatcher' with $L r 34$ exhibits resistance.

In this report, evidence is presented that, in a 'Canthatch' background, Lr34 inactivates the suppressor on chromosome arm 7DL

Corresponding author: T. Aung; E-mail address: taung@em.agr.ca

Publication no. P-1999-0427-03R

(c) 1999 Department of Agriculture and Agri-Food, Government of Canada and thereby permits the expression of resistance genes normally inhibited by the suppressor.

\section{MATERIALS AND METHODS}

To determine whether the presence of Lr34 would result in resistance to specific races of stem rust otherwise inhibited by the 7DL suppressor, chromosome 7D of 'Canthatch' was substituted by the corresponding chromosome of the cultivar Chinese Spring known to possess $\operatorname{Lr} 34$ (2). 'Chinese Spring' was selected as the source of Lr34, located on 7DS, because this cultivar as well as the 7DS ditelosomic are susceptible to the relevant races of stem rust. A stock true for 'Chinese Spring' ditelosomic 7DL was unavailable for testing. If $L r 34$ or a linked gene on 'Chinese Spring' chromosome 7D behaves as a nonsuppressor, the substitution line should exhibit resistance comparable to 'Canthatch' nullisomic 7D.

Chromosome 7D of 'Canthatch' was substituted by 7D of 'Chinese Spring' by crossing 'Canthatch' nullisomic 7D $(2 n=40)$ with 'Chinese Spring' $(2 \mathrm{n}=42)$, followed by four successive backcrosses of the $F_{1}$ monosomic $(2 n=41)$, as the male parent, to the 'Canthatch' nullisomic recurrent parent. On completion of the last backcross in the development of the "Canthatch'5-7D/"Chinese Spring' substitution, spikes of the cytologically confirmed monosomic $F_{1}$ plants were bagged to provide seed for tests of the backcross $F_{2}$ with stem rust. Sets of approximately $50 \mathrm{~F}_{2}$ seedlings from each of six $F_{1}$ monosomic substitution plants were grown in the greenhouse and inoculated at the one- to two-leaf stage with stem rust races QTH and RTH. Previously, these races were designated as C25 and C57, respectively $(9,10,11)$. 'Canthatch' is susceptible to these as well as other races because of the dominant suppressor located on 7DL $(10,11)$. The virulence formulas of QTH, RTH, and additional races to which the experimental material was tested are given in Table $1 . \mathrm{F}_{2}$ plants were selected that gave the same resistant reaction to QTH and RTH as did 'Canthatch' nullisomic 7D and were cytologically examined for chromosome constitution. These selections were progeny-tested with both races. Chromosome pairing was examined at metaphase I in pollen 
mother cells (PMCs) of the initial $\mathrm{F}_{1}$ hybrid 'Canthatch' nullisomic 7D $\times$ 'Chinese Spring' and the final backcross $\mathrm{F}_{1}$ of 'Canthatch' nullisomic 7D $\times$ 'Chinese Spring'. For comparative purposes, the tests with races QTH and RTH included 'Chinese Spring'; 'Canthatch' nullisomic 7D; 'Chinese Spring' ditelosomic 7DS; 'Canthatch'; 'Canthatch'-NS, which possesses a nonsuppressing mutation on chromosome 7DL (9); 'Thatcher'; 'RL6058', a 'Thatcher' backcross derivative in which the leaf rust resistance gene $L r 34$ confers enhanced expression of resistance to races QTH and RTH (1); 'Tetracanthatch' $(2 \mathrm{n}=28$; AABB $)$, the extracted AABB tetraploid component of 'Canthatch' (8) that is resistant to races QTH and RTH; 'Canthatch' ditelosomic 7DL, which is susceptible to QTH and RTH because of the suppressor gene on this telosomic (9); and 'Canthatch' ditelosomic 7DS, which is resistant to these two races because of the absence of the 7DL suppressor (9). Seedling infection types to QTH, RTH, and several other relevant races are given in Table 2 for the stocks described above. Also presented is the field reaction of some of the stocks to a composite of eight races.

Procedures for rust inoculation, scoring infection type (20), and cytological analyses were similar to those previously employed (9). The nomenclature of the stem rust races is according to Roelfs and Martens (16). The parental material and related genetic stocks used in the investigation are listed in Table 2.

\section{RESULTS}

If leaf rust resistance gene $L r 34$ permits the expression of suppressed resistance genes in 'Canthatch', the substitution of chromosome 7D of this cultivar by 7D of 'Chinese Spring' should

TABLE 1. Virulence formulas of stem rust races

\begin{tabular}{ll}
\hline Race $^{\mathrm{y}}$ & \multicolumn{1}{c}{ Effective/ineffective stem rust resistance genes } \\
\hline QTH & 7b,9a,9e,30,36/5,6,7a,8a,9b,9d,9g,10,11,12,13,14,15,16,17,21 \\
RTH & $9 \mathrm{a}, 9 \mathrm{e}, 30,36 / 5,6,7 \mathrm{a}, 7 \mathrm{~b}, 8 \mathrm{a}, 9 \mathrm{~b}, 9 \mathrm{~d}, 9 \mathrm{~g}, 10,11,12,13,14,15,16,17,21$ \\
RKQ $^{\mathrm{z}}$ & $9 \mathrm{e}, 10,11,13,17 / 5,6,7 \mathrm{a}, 7 \mathrm{~b}, 8 \mathrm{a}, 9 \mathrm{a}, 9 \mathrm{~b}, 9 \mathrm{~d}, 14,15,36$ \\
RKQ & $7 \mathrm{a}, 9 \mathrm{e}, 10,11,13,17 / 5,6,7 \mathrm{~b}, 8 \mathrm{a}, 9 \mathrm{a}, 9 \mathrm{~b}, 9 \mathrm{~d}, 14,15,36$ \\
TPM & $6,9 \mathrm{a}, 9 \mathrm{~b}, 13,15 / 5,7 \mathrm{a}, 7 \mathrm{~b}, 8 \mathrm{a}, 9 \mathrm{~d}, 9 \mathrm{e}, 10,11,14,17,36$ \\
MCC & $6,8,9 \mathrm{a}, 9 \mathrm{~b}, 9 \mathrm{~d}, 9 \mathrm{e}, 11,13,36 / 5,7 \mathrm{a}, 7 \mathrm{~b}, 10,14,15,17$ \\
HFH & $5,6,9 \mathrm{a}, 9 \mathrm{e}, 11,36 / 7 \mathrm{a}, 7 \mathrm{~b}, 8 \mathrm{a}, 9 \mathrm{~b}, 9 \mathrm{~d}, 10,13,14,15,17$ \\
\hline
\end{tabular}

y Stem rust race Puccinia graminis f. sp. tritici nomenclature (16).

$\mathrm{z}$ This culture of race RKQ was virulent on all genetic stocks tested (Table 2). confer resistance to races QTH and RTH comparable to that of 'Canthatch' nullisomic 7D (IT 0;) (Table 2). The progenies of $\mathrm{F}_{1}$ monosomic $(2 \mathrm{n}=41)$ substitution plants of the third ('Canthatch'$7 \mathrm{D}^{4} /{ }^{\prime}$ Chinese Spring') and fourth ('Canthatch'-7D $5 /$ 'Chinese Spring') backcrosses (backcross $\mathrm{F}_{2}$ ) segregated to QTH and RTH. Segregation resulted from the expression of a resistance gene(s) contributed by a chromosome(s) from 'Canthatch' other than 7D. Numerous plants exhibiting IT 0; were selected. The chromosome constitution of these was $2 n=42,2 n=41$, or $2 n=40$. Thus, the full resistance was expressed in the disomic and the monosomic 7D (hemizygous effective) substitution, as well as the nullisomic. Additional tests on lines established in $\mathrm{F}_{3}$ to $\mathrm{F}_{5}$ that were homozygous resistant to either QTH or RTH indicated that all were equally resistant to both races, regardless of the chromosome 7D constitution. Consequently, 'Chinese Spring' chromosome 7D, possessing Lr34 on the short arm, permitted expression of previously inhibited resistance genes of unknown identity when substituted for the corresponding chromosome of 'Canthatch'. Resistant nullisomic plants $(2 n=40)$ would be expected in these monosomic progenies, since, in the absence of chromosome 7D, the 'Canthatch' nullisomic itself is resistant.

As expected, 'Canthatch' ditelosomic 7DS (IT 0;) and 'Tetracanthatch' (IT 0;), which lack the 7DL suppressor, as well as 'Canthatch'-NS (IT 0;), which possesses a nonsuppressing mutant allele on chromosome 7DL, were similar in reaction to 'Canthatch' nullisomic 7D (Table 2). On the other hand, 'Canthatch' (IT 32+) and 'Canthatch' ditelosomic 7DL (IT 32+) were moderately susceptible to races QTH and RTH because of the presence of the suppressor on 7DL. 'Chinese Spring' and 'Chinese Spring' ditelosomic 7DS were fully susceptible (IT $3^{+} 4$ ) because they lack resistance to the races tested, regardless of the presence of $L r 34$. The resistance of 'Thatcher'-Lr34 ('RL6058') (IT 0;) was comparable to that of 'Canthatch' nullisomic 7D, confirming previous evidence $(1,3,6)$ that, in a 'Thatcher' background, Lr34 enhances resistance relative to 'Thatcher'. The results of these tests with races QTH and RTH were corroborated by seedling tests with additional races and by field tests of relevant stocks to a composite of races. Notably, however, a culture of race RKQ was virulent on all stocks at the seedling stage (Table 2).

To establish that chromosome 7D of 'Canthatch' had indeed been substituted by an intact 7D of 'Chinese Spring', chromosome pairing at metaphase $\mathrm{I}$ was examined in PMCs of $\mathrm{F}_{1}$ monosomic substitution plants of the initial cross 'Canthatch' nullisomic 7D $\times$

TABLE 2. Chromosome number, seedling infection type to several races of stem rust, and field reaction of homozygous resistant lines of disomic $(2 \mathrm{n}=42)$ and monosomic $(2 \mathrm{n}=41)$ substitutions of 'Canthatch'-7D/“Chinese Spring' and of related genetic stocks

\begin{tabular}{|c|c|c|c|c|c|c|c|c|c|}
\hline \multirow[b]{2}{*}{ Genetic stock } & \multirow{2}{*}{$\begin{array}{c}\text { Chromosome } \\
\text { number }\end{array}$} & \multicolumn{7}{|c|}{ Race of rust ${ }^{\mathrm{u}}$ and infection type $\mathrm{v}^{\mathrm{v}}$} & \multirow{2}{*}{$\begin{array}{l}\text { Field } \\
\text { reaction }{ }^{\mathrm{w}}\end{array}$} \\
\hline & & QTH & RTH & $\mathrm{RKQ}^{\mathrm{x}}$ & RKQ & $\mathrm{MCC}$ & TPM & $\mathrm{HFH}$ & \\
\hline 'Canthatch'-7D/‘Chinese Spring' & 42,41 & 0 & 0 & $3^{+}$ & 0 & 0 & 0 & 0 & $\mathrm{TR}$ \\
\hline 'Chinese Spring' & 42 & $3+4$ & $3+4$ & $\ldots$ & $\ldots$ & $\ldots$ & $\ldots$ & $\ldots$ & $60 \mathrm{~S}$ \\
\hline 'Canthatch' & 42 & $32+y$ & $32^{+}$ & $3^{+}$ & $2^{-}$ & $; 1$ & $3^{+}$ & 0 & 25 MR-MS \\
\hline 'Canthatch’ nullisomic 7D & 40 & 0 & 0 & $3^{+}$ & 0 & 0 & 0 & 0 & $5 \mathrm{R}$ \\
\hline 'Thatcher'-Lr34 ('RL6058') & 42 & 0 & 0 & $3^{+}$ & 0 & 0 & 0 & 0 & $\mathrm{TR}$ \\
\hline 'Thatcher' & 42 & $32^{+}$ & $32^{+}$ & $3^{+}$ & $2+3$ & $; 1$ & $3^{+}$ & 0 & $30 \mathrm{MS}$ \\
\hline 'Canthatch'-NS & 42 & 0 & 0 & $\ldots$ & $\ldots$ & $\ldots$ & $\ldots$ & $\ldots$ & $5 \mathrm{R}$ \\
\hline 'Tetracanthatch' & 28 & 0 & 0 & $\ldots$ & $\ldots$ & $\ldots$ & $\ldots$ & $\ldots$ & $\ldots$ \\
\hline 'Chinese Spring' ditelosomic 7DS & $40+2 t^{\mathrm{z}}$ & $3^{+}$ & $3^{+}$ & $\ldots$ & $\ldots$ & $\ldots$ & $\ldots$ & $\ldots$ & $\ldots$ \\
\hline 'Canthatch' ditelosomic 7DS & $40+2 t$ & 0 & 0 & $3^{+}$ & 0 & 0 & 0 & 0 & $\ldots$ \\
\hline 'Canthatch' ditelosomic 7DL & $40+2 t$ & $3^{+} 2^{+}$ & $3^{+} 2^{+}$ & $3^{+}$ & $2^{-}$ & $; 1$ & $3^{+}$ & 0 & $\ldots$ \\
\hline
\end{tabular}

u Stem rust race Puccinia graminis f. sp. tritici nomenclature (16).

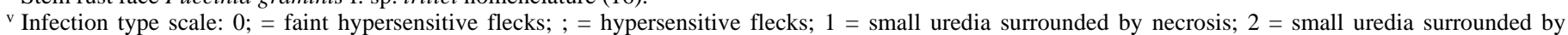
chlorosis; 3 = moderate size uredia without chlorosis; $+=$ slightly larger uredia than expected for the infection type; $-=$ slightly smaller uredia than expected for the infection type; and $\ldots=$ not tested.

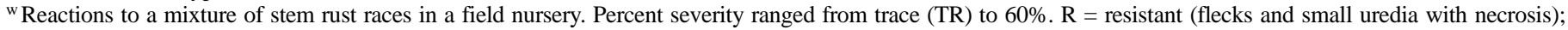
$\mathrm{MR}=$ moderately resistant (large necrotic flecks and uredia); MS = moderately susceptible (moderate to large uredia with chlorosis); $\mathrm{S}=$ susceptible (large uredia); and $\ldots=$ not tested.

x This culture of race RKQ was virulent on all genetic stocks tested.

${ }^{y}$ First mentioned infection type most prevalent.

${ }^{\mathrm{z}} \mathrm{t}=$ Telocentric chromosome. 
TABLE 3. Chromosome pairing in metaphase I pollen mother cells of monosomic $F_{1}$ plants $(2 n=41)$ of substitution crosses 'Canthatch' nullisomic-7D/'Chinese Spring' and 'Canthatch' nullisomic-7D $/ \mathrm{D}^{5}$ Chinese Spring'

\begin{tabular}{|c|c|c|c|c|c|c|}
\hline \multirow[b]{2}{*}{ Cross } & \multirow[b]{2}{*}{ No. of plants } & \multicolumn{4}{|c|}{ Chromosome pairing ${ }^{y}$ and no. of cells } & \multirow[b]{2}{*}{ Total no. of cells } \\
\hline & & $20^{\mathrm{ii}}+1^{\mathrm{i}}$ & $19^{\mathrm{ii}}+3^{\mathrm{i}}$ & $18^{\mathrm{ii}}+1^{\mathrm{iv}}+1^{\mathrm{i}}$ & $18^{\mathrm{ii}}+1^{\mathrm{iii}}+2^{\mathrm{i}}$ & \\
\hline 'Canthatch’ nullisomic-7D/“Chinese Spring' & 3 & $234(82.4)^{\mathrm{z}}$ & $39(13.7)$ & $8(2.8)$ & $3(1.1)$ & 284 \\
\hline 'Canthatch' nullisomic-7D $5 /$ 'Chinese Spring' & 4 & $343(85.8)$ & $13(3.3)$ & $39(9.8)$ & $5(1.3)$ & 400 \\
\hline
\end{tabular}

y Accepted cytogenetic designation of chromosome pairing in individual cells. $i=$ Univalent; ii = bivalent; iii = trivalent; and iv = quadrivalent.

${ }^{\mathrm{z}}$ Percent cells in brackets.

'Chinese Spring' and of the final backcross 'Canthatch' nullisomic $7 \mathrm{D}^{5} \times$ 'Chinese Spring' (Table 3). Although 3.9\% of the PMCs of the initial hybrid and $11.1 \%$ of the last backcross $F_{1}$ possessed a multivalent (either a quadrivalent or a trivalent plus a univalent), all had at least one univalent in those cells without a multivalent or with one quadrivalent and had two univalents in cells with a trivalent. 'Canthatch' and 'Chinese Spring' are known to differ by a translocation (E. R. Kerber, unpublished data). The metaphase I pairing associations in these $F_{1}$ populations conform with the conclusion that chromosome 7D of 'Chinese Spring' occurred as an intact univalent in the initial hybrid and that this monosome replaced the corresponding homologue of 'Canthatch' in the substitution stock produced at the completion of the final backcross. The pairing data indicate that chromosome 7D was not involved in a translocation or other meiotic irregularity in the hybrid material examined.

\section{DISCUSSION}

The cytogenetic and pathologic evidence obtained in the current investigation confirms and clarifies Dyck's $(1,3)$ finding that, in a 'Thatcher' background, leaf rust resistance gene Lr34 is associated with enhanced stem rust resistance (Table 2). The 'Thatcher' backcross derivative possessing Lr34 ('RL6058') on the short arm of chromosome 7D exhibits the same resistant reaction (IT 0;) to races QTH and RTH as does 'Canthatch' nullisomic 7D, whereas 'Thatcher' is moderately susceptible (IT 32+). Furthermore, 'Thatcher'-Lr34 presumably also carries the 7DL suppressor (as in 'Canthatch') since it, like 'Canthatch', is a backcross derivative of 'Thatcher'. Consequently, Lr34 likely inactivates the suppressor. Our experimental results also demonstrate that substitution of chromosome 7D of 'Canthatch' by 7D of 'Chinese Spring' ('Canthatch'-7D/'Chinese Spring') permits expression of resistance to races QTH and RTH to which 'Canthatch' is moderately susceptible. It is not apparent, however, whether the nonsuppressing effect of 'Chinese Spring' chromosome 7D is due (i) directly to Lr34 (or linked gene), (ii) to the absence of a suppressor on the long arm, or (iii) to the inactivation by Lr34 of a suppressor on this arm. The latter is the most plausible interpretation in that interaction between Lr34 (short arm) and an assumed suppressor (long arm) would be similar to that suggested for 7D of 'Thatcher'-Lr34. Overall, our results indicate that neither the 'Thatcher'-Lr34 nor 'Chinese Spring' chromosome 7D possesses a nonsuppressing allele of the 7DL suppressor.

It would be of importance to determine the number of resistance genes not expressed in 'Canthatch' because of the 7DL suppressor. Tentative evidence (21) indicated that three or more recessive genes are inhibited. Similarly, segregation of $\mathrm{F}_{3}$ families of the cross 'Pelissier' durum wheat (susceptible) $\times$ 'Tetracanthatch' (resistant) also suggested that three recessive genes could be involved (E. R. Kerber, unpublished data). 'Tetracanthatch' is the extracted AABB component of 'Canthatch' that exhibits the same resistant reaction to races QTH and RTH as does 'Canthatch' nullisomic 7D (IT 0;). It appears, therefore, that the 7DL suppressor inhibits more than one resistance gene of unknown identity; it is not resistance-gene specific.

Lr34 was initially identified and characterized as a leaf rust resistance gene effective primarily at the adult-plant stage (6). Since then, several characters and distinctive traits have been found to be associated with or attributed to this locus. The gene $\operatorname{Yrl} 18$ for resistance to stripe rust (P. striiformis Westend.) and the gene $B d v 1$ for resistance to barley yellow dwarf virus are closely linked to or result from pleiotropic effects of Lr34 (19). Lr34 is associated with the gene $L t n$, which induces leaf tip necrosis and thereby serves as a marker to identify the presence of $L r 34, \operatorname{Yr} 18$, and $B d v 1$ (18). $L r 34$ can facilitate the interspecific transfer of leaf rust resistance genes from a lower to a higher level of ploidy. For example, resistance was transferred to hexaploid wheat from diploid $(2 \mathrm{n}=14 ; \mathrm{AA})$ and tetraploid $(2 \mathrm{n}=28 ; \mathrm{AABB})$ wheats by the use of 'Thatcher'-Lr34 as the recipient parent (4). A significant attribute of $L r 34$ is the enhancement of resistance exhibited when it is combined with specific other leaf rust resistance genes; the resistance expressed is greater than that when the combined genes are present singly $(7,12,17)$. Of direct bearing on the current investigation is the observation by Dyck $(1,3)$ that, in 'Thatcher' and 'Thatcher' backcross-derived cultivars, Lr34 or associated chromatin enhances resistance to stem rust. Our results confirm this finding and provide pathologic and cytogenetic evidence to account for this attribute. The presence of $\mathrm{Lr} 34$ most likely permits the expression of inhibited resistance genes by inactivating the 7DL suppressor.

From a plant breeding viewpoint, the increased stem rust resistance in cultivars of 'Thatcher' parentage possessing Lr34 is noteworthy. Kolmer (12) states that, of the numerous Canadian and American hard red spring wheat cultivars derived from 'Thatcher', those possessing Lr34 could have a significant share of their stem rust resistance attributable to this gene. For example, the Canadian cultivars Roblin and Pasqua, both having Lr34, were noted to exhibit resistance greater than that expected from the known resistance genes possessed by each $(3,13)$. We are unaware whether stem rust resistance is improved by the presence of Lr34 in cultivars other than 'Thatcher' and closely related backcross derivatives.

\section{ACKNOWLEDGMENTS}

We thank K. Dunsmore for valuable technical assistance.

\section{LITERATURE CITED}

1. Dyck, P. L. 1987. The association of a gene for leaf rust resistance with the chromosome 7D suppressor of stem rust resistance in common wheat. Genome 29:467-469.

2. Dyck, P. L. 1991. Genetics of adult-plant leaf rust resistance in 'Chinese Spring' and 'Sturdy' wheats. Crop Sci. 31:309-311.

3. Dyck, P. L. 1993. Inheritance of leaf rust and stem rust resistance in 'Roblin' wheat. Genome 36:289-293.

4. Dyck, P. L., and Bartoš, P. 1994. Attempted transfer of leaf rust resistance from Triticum monococcum and durum wheat to hexaploid wheat. Can. J. Plant Sci. 74:733-736.

5. Dyck, P. L., Kerber, E. R., and Aung, T. 1994. An interchromosomal reciprocal translocation in wheat involving leaf rust resistance gene Lr34. Genome 37:556-559.

6. Dyck, P. L., and Samborski, D. J. 1982. The inheritance of resistance to Puccinia recondita in a group of common wheat cultivars. Can. J. Genet. Cytol. 24:273-283.

7. German, S. E., and Kolmer, J. A. 1992. Effect of gene Lr34 in the enhancement of resistance to leaf rust of wheat. Theor. Appl. Genet. 84:97-105.

8. Kerber, E. R. 1964. Wheat: Reconstitution of the tetraploid component (AABB) of hexaploids. Science 143:253-255.

9. Kerber, E. R. 1991. Stem rust resistance in 'Canthatch' hexaploid wheat 
induced by a nonsuppressor mutation on chromosome 7DL. Genome 34: 935-936.

10. Kerber, E. R., and Aung, T. 1995. Confirmation of nonsuppressor mutation of stem rust resistance in 'Canthatch' common wheat. Crop Sci. 35: 743-744.

11. Kerber, E. R., and Green, G. J. 1980. Suppression of stem rust resistance in the hexaploid wheat cv. Canthatch by chromosome 7DL. Can. J. Bot. 58:1347-1350.

12. Kolmer, J. A. 1996. Genetics of resistance to wheat leaf rust. Annu. Rev. Phytopathol. 34:435-455.

13. Liu, J. Q., and Kolmer, J. A. 1998. Genetics of stem rust resistance in wheat cvs. Pasqua and AC Taber. Phytopathology 88:171-176.

14. McIntosh, R. A., Hart, G. E., and Gale, M. D. 1993. Catalogue of gene symbols for wheat. Page 1408 in: Proc. Int. Wheat Genet. Symp., 8th. Vol. 2. Z. S. Li and Z. Y. Xin, eds. China Agricultural Scientech Press, Beijing.

15. Nelson, J. C., Singh, R. P., Autrique, J. E., and Sorrells, M. E. 1997. Mapping genes conferring and suppressing leaf rust resistance in wheat.
Crop Sci. 37:1928-1935.

16. Roelfs, A. P., and Martens, J. W. 1988. An international system of nomenclature for Puccinia graminis f. sp. tritici. Phytopathology 78:526-533.

17. Samborski, D. J., and Dyck, P. L. 1982. Enhancement of resistance to Puccinia recondita by interactions of resistance genes in wheat. Can. J. Plant Pathol. 4:152-156.

18. Singh, R. P. 1992. Association between gene Lr34 for leaf rust resistance and leaf tip necrosis in wheat. Crop Sci. 32:874-878.

19. Singh, R. P. 1993. Genetic association of gene $B d v 1$ for tolerance to barley yellow dwarf virus with genes $\operatorname{Lr} 34$ and $\mathrm{Yr} 18$ for adult plant resistance to rusts in bread wheat. Plant Dis. 77:1103-1106.

20. Stakman, E. C., Stewart, D. M., and Loegering, W. Q. 1962. Identification of physiologic races of Puccinia graminis var. tritici. USDA-ARS E67. Sci. J. Ser. Paper No. 4691. Minnesota Agric. Exp. Stn., St. Paul, MN.

21. Williams, N. D., Miller, J. D., and Klindworth, D. L. 1992. Induced mutations of a genetic suppressor of resistance to wheat stem rust. Crop Sci. 32:612-616. 